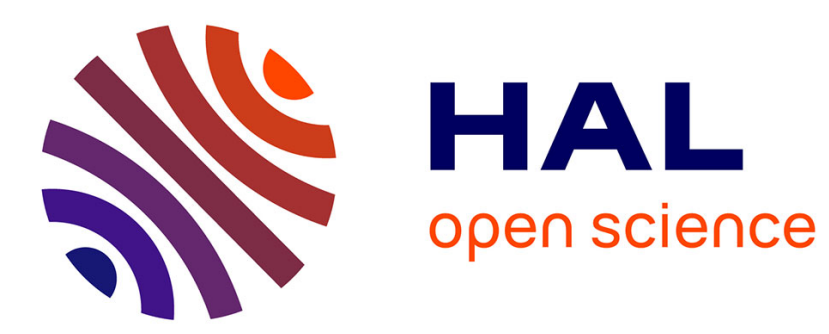

\title{
Full solution to the mean spherical approximation (MSA) for an arbitrary mixture of ions in a dipolar solvent
}

Jean-Pierre Simonin, Johan S Høye

\section{- To cite this version:}

Jean-Pierre Simonin, Johan S Høye. Full solution to the mean spherical approximation (MSA) for an arbitrary mixture of ions in a dipolar solvent. Journal of Chemical Physics, 2021, 155 (11), pp.114502. 10.1063/5.0064188 . hal-03380313

\section{HAL Id: hal-03380313 \\ https://hal.sorbonne-universite.fr/hal-03380313}

Submitted on 15 Oct 2021

HAL is a multi-disciplinary open access archive for the deposit and dissemination of scientific research documents, whether they are published or not. The documents may come from teaching and research institutions in France or abroad, or from public or private research centers.
L'archive ouverte pluridisciplinaire HAL, est destinée au dépôt et à la diffusion de documents scientifiques de niveau recherche, publiés ou non, émanant des établissements d'enseignement et de recherche français ou étrangers, des laboratoires publics ou privés. 


\title{
Full solution to the mean spherical approximation (MSA) for an arbitrary mixture of ions in a dipolar solvent ${ }^{\text {a) }}$
}

\author{
Jean-Pierre Simonin ${ }^{1}$ and Johan S. Høye ${ }^{2}$ \\ 1) Laboratoire PHENIX, CNRS, Sorbonne Université (Campus P.M. Curie), \\ 4 Place Jussieu, Case 51, F-75005, Paris, France ${ }^{\mathrm{b})}$ \\ 2) Department of Physics, Norwegian University of Science and Technology (NTNU), \\ Norway ${ }^{c)}$
}

The complete solution to the mean-spherical approximation is given for an arbitrary mixture of ions in a dipolar solvent. The calculation is done for an overall neutral collection of charged hard spheres (the ions), and dipoles (the solvent) that bear a central point dipole. Previous solutions to this problem were not consistent because they did not include the solvent particles as a (neutral) solute in the mixture. The present solution accounts for this peculiar counterintuitive feature, fully and consistently. Complete expressions for the chemical potentials of the ions and of the solvent are provided for the first time. To illustrate, a binary 1-1 asymmetric electrolyte solution in a water-like dipolar solvent is considered, and some numerical results are examined. In particular, it is verified that a set of thermodynamic consistency relations are accurately fulfilled.

Keywords: Electrolytes; non-primitive; mean-spherical approximation (MSA); unrestricted case; dipoles.

\footnotetext{
a) This work is dedicated to the memory of L. Blum, a colleague and friend.

b) E-mail: jpsimonin@gmail.com

c)E-mail: johan.hoye@ntnu.no
} 


\section{INTRODUCTION}

The system comprised of ions and dipoles was first approached by L. Blum in $1978^{1}$ within the mean spherical approximation (MSA). In this MSA model for ions and dipoles (hereafter denoted as MSA-ID), the ions are represented as charged hard spheres, and the dipoles, which may mimic water molecules, are hard spheres bearing a central point dipole. This system is sketched in Figure 1.

Blum used an involved invariant expansion formalism ${ }^{2}$ to account for angular correlations involving the dipoles. In subsequent work, the thermodynamic properties of the MSA-ID model were studied by Blum and $\mathrm{Wei}^{3,4}$.

It was found recently that the reported complicated equations contained some misprints, that were corrected in $2020^{5}$. However, a further closer examination of the corrected formulas revealed lately that a thermodynamic consistency relation involving the pressure was not fulfilled. Moreover, as will be seen below, we recently found that some of the equations did not allow one to recover the (primitive) MSA equations obtained when the dipole moment of the solvent is set to zero.

It was finally realized that this observation imposed to not only consider a solvent particle as a hard dipole, but also to regard it as a solute (a "neutral" ion), on the same footing as the ions comprising the electrolyte. This dual character of the dipoles has been overlooked in previous treatments within the MSA. However, this new viewpoint implies the need for introducing new, somewhat counterintuitive or "unnatural", parameters for the solvent in the description.

The structure of this article is as follows. In the next section, some problems observed with the available equations for the MSA-ID model are listed. Then, a solution to these problems is proposed, and new equations for the MSA-ID model are presented. Modified relations for the thermodynamic quantities are deduced accordingly in the fourth section. Particular attention is devoted to the derivation of the chemical potentials of the species. A suitable method is developed for the calculation of the chemical potential of the solvent. The validity of the solution is tested by examining the numerical accuracy of several thermodynamic consistency relations. These checks show that the present work provides the full solution to the MSA-ID problem. The fifth section is devoted to an illustration, in which a binary electrolyte of a 1-1 salt in a dipolar water-like solvent is considered. The set of 
MSA equations is solved numerically to obtain the solution for a particular case, and the consistency of the equations is verified for this solution. The main features exhibited by the thermodynamic quantities are analyzed. Finally, the results are summarized and some prospects are presented in the conclusion section.

\section{MSA-ID MODEL: PROBLEM TO BE SOLVED}

In what follows, Ref. 1 will be denoted by B0, Ref. 3 by BW1, Ref. 4 by BW2, Ref. 6 by BVF, and Ref. 5 by S0.

\section{A. The model}

We consider a mixture of hard spherical cations and anions, and hard spheres with a centrally embedded point dipole (the solvent), as a model of electrolyte solution. The purpose of the present study is the solution of the MSA for this type of system in which all species may have different sizes.

As in B0, BW1, BW2, and S0 the number of ions will be denoted by $n-1$, and the solvent by index $n$. The dipole moment of the solvent particles is $d_{n}$. The valence and diameter of an ion $i$ are $Z_{i}$, and $\sigma_{i}$, respectively. A priori, the diameters $\sigma_{k}(k=1, \ldots, n)$ are all different.

In B0, L. Blum presented for the first time an elaborate method to solve the MSA-ID model for the case of ions and solvent of arbitrary size, that involved the use of rotational invariants $^{2,7,8}$. This tool allowed him to describe properly the orientation-dependent character of the ion-dipole and dipole-dipole interactions. The basic features of the framework employed by Blum were presented in S0. As in the latter, the notations of B0 are kept in the present work.

\section{B. Observed issues in the available equations}

Before giving the correct equations of the model, the reasons that suggested that the available solution to the MSA-ID was incomplete are now exposed.

After the publication of S0 in 2020, it was found that some thermodynamic consistency relations were not accurately fulfilled. For example, one of them was (other relations are 
examined below in Section IV D),

$$
\frac{\partial \beta P}{\partial \beta}=-\frac{\partial E}{\partial V}
$$

where $P$ is the excess electrostatic pressure, $\beta=1 / k_{B} T$ (with $k_{B}$ the Boltzmann constant and $T$ the temperature), $E$ is the excess electrostatic internal energy, and $V$ is the volume.

The fact that this relation was (slightly) unfulfilled showed that the pressure was (presumably slightly too) incorrect, because once the MSA parameters have been determined (see S0), then $E$ should be known without ambiguity. It was therefore concluded that something was missing in the approach.

This conclusion was realized and by that reinforced when looking more closely at the case where the dipole moment on the solvent particles is set to zero $\left(d_{n}=0\right)$. In that particular case, $n$ becomes a neutral solute and the solution to the MSA for an asymmetric electrolyte in the primitive model ${ }^{9,10}$ should be recovered. However, surprisingly enough, it was observed that this was not the case. This anomaly may be seen for instance in Eq. (15) of S0 in which $a_{n}^{1}=0$ when $d_{n}=0$ (see its Eq. (20)) because in that case, all $m_{i}$ 's vanish ( $m_{i}$ is a parameter for the ion-dipole interaction). Then, it is seen that a term involving $a_{n}^{0}$ is missing in this fundamental equation. A similar anomaly was also noticed in Eq. (24) of S0 for $D_{a c}$, which is denoted by $D$ in the primitive MSA (see, e.g., Eq. (B.45) of Ref. 11) and includes the neutral solute $k=n$ as any other species in the mixture.

Another intriguing point was noticed in Eqs. (35) and (44) of S0 for two types of iondipole parameters which, contrary to similar parameters for ion-ion interactions, do not contain any electrostatic contribution.

On the basis of these observations it was hypothesized that the solvent should also be regarded as a solute species in all equations, for the particular case $d_{n}=0$ to be taken into account properly in the model, and for the primitive MSA equations to be fully recovered.

However, this raised an unexpected problem because, by doing so, the dipolar particles acquired a dual status of being both a solvent and a solute particle. Technically, this implies that new MSA parameters should be introduced by which the $n$ particles are also regarded as a solute.

This new angle was somewhat disturbing because, in all former approaches, the system was, naturally enough, divided into two separate constituents, namely the solute species and the solvent. At the same time, the above considerations suggested a way for solving the problem. Indeed, it was observed in S0 that the apparently incomplete equations nonetheless 
formed an internally consistent set of relations (see, e.g., the results of section B.1 of S0). Therefore it was reasonably expected that considering also the solvent as a solute could yield a set of consistent equations if the modifications were done in a coherent way. Then, it was hypothesized that a natural solution could be to simply introduce the solvent $n$ as an extra solute particle in all available equations, in particular in those where a sum over the solute species is performed.

This is what is done in the next section, in which the modified equations are given.

\section{SOLUTION OF THE MSA-ID MODEL}

The equations of the MSA-ID model were taken from S0 and corrected according to the indications of the preceding section.

Let us note for clarity that, hereafter, in any symbol of the form $M_{i j}^{01}$ for example, $i$ may represent an ion or a solvent particle because it is located below the superscript "0", and that $j$ necessarily designates a solvent particle $(j=n)$ because it is located below the superscript " 1 ".

The first three fundamental equations (Eqs. (1.29), (1.30) and (1.32) of BW1) are,

$$
\begin{gathered}
\sum_{i=1}^{n} \rho_{i}\left(a_{i}^{0}\right)^{2}+\rho_{n}\left(a_{n}^{1}\right)^{2}=\alpha_{0}{ }^{2}, \\
-\sum_{j=1}^{n} \rho_{j} a_{j}^{0} K_{n j}^{10}+a_{n}^{1}\left(1-\rho_{n} K_{n n}^{11}\right)=\alpha_{0} \alpha_{2}, \\
\rho_{n} \sum_{j=1}^{n} \rho_{i}\left(K_{n j}^{10}\right)^{2}+\left(1-\rho_{n} K_{n n}^{11}\right)^{2}=y_{1}{ }^{2}+\rho_{n} \alpha_{2}{ }^{2},
\end{gathered}
$$

In these relations, the new parameters $a_{n}^{0}$ and $K_{n n}^{10}$ are introduced for the first time. In previous treatments, $a_{i}^{0}$ was a parameter for solute species exclusively. As mentioned at the beginning of this section, the superscript " 0 " in $K_{n n}^{10}$ means that the second solvent particle $n$ in the subscript is regarded as a solute. Conversely, the superscript " 1 " in $K_{n n}^{10}$ indicates that the first solvent particle $n$ in the subscript is regarded as a dipolar species. It may be mentioned that the introduction of these parameters and the extension of the summations to include $i, j=n$ also follow from the graph analysis of the MSA problem performed in Sec. IV B 3 below. 
In Eqs. (2)-(4), $\rho_{i}$ is the number density of species $i$ (number of particles $i$ per volume unit), and,

$$
\alpha_{0}^{2}=\beta e^{2} / \epsilon_{0}, \quad \alpha_{2}{ }^{2}=\beta d_{n}{ }^{2} /\left(3 \epsilon_{0}\right), \quad y_{1}=\beta_{6} / \beta_{12}{ }^{2},
$$

with $\beta=1 /\left(k_{B} T\right)$ (with $T$ the temperature and $k_{B}$ the Boltzmann constant), $e$ is the charge of a proton, $\varepsilon_{0}$ is the permittivity of a vacuum, and

$$
\beta_{6}=1-b_{2} / 6, \quad \beta_{12}=1+b_{2} / 12
$$

with $b_{2}$ a dimensionless parameter characteristic of the dipole-dipole interaction ${ }^{6}$.

Furthermore, $a_{i}^{0}$ (for $\left.i=1, \ldots, n\right)$ and $a_{n}^{1}$ (in which the superscript "1" indicates the dipolar character of $n$ in this case) are given by,

$$
a_{i}^{0}=\frac{\beta_{6}}{\sigma_{i} D_{a}}\left(\frac{1}{2} Z_{i} \beta_{6}-D_{i}^{G}-\frac{L}{2} \sigma_{i}^{2} S_{G}\right)
$$

with,

$$
L=\frac{\pi}{\Delta}, \quad \Delta=1-\frac{\pi}{6} \sum_{k=1}^{n} \rho_{k} \sigma_{k}{ }^{3} \quad S_{G}=\sum_{k=1}^{n} \rho_{k} \sigma_{k} D_{k}^{G},
$$

in which $\Delta$ is the fraction of free space. Moreover,

$$
a_{n}^{1}=\frac{\beta_{6}}{2 D_{a}}\left(\frac{1}{2} \sigma_{n} B+\frac{\Omega \beta_{3}}{D \beta_{6}^{2}}\right)
$$

with $\beta_{3}=1+b_{2} / 3$, and,

$$
\begin{gathered}
B=\sum_{k=1}^{n-1} \rho_{k} Z_{k} m_{k}, \quad D=1+\frac{\rho_{n} \sigma_{n}{ }^{2}}{4 \beta_{6}{ }^{2}} \sum_{k=1}^{n} \rho_{k}\left(\sigma_{k} m_{k}\right)^{2}, \\
\eta_{i}=\frac{1}{\beta_{6}}\left(m_{i}+\sigma_{i} S_{m}\right), \quad S_{m}=\frac{L}{2} \sum_{k=1}^{n} \rho_{k} \sigma_{k}{ }^{2} m_{k}, \\
D_{k}^{F}=\frac{1}{2}\left[\beta_{6}\left(N_{k} \sigma_{k}+Z_{k}\right)-\frac{1}{12} \rho_{n} \sigma_{n}{ }^{3} B \sigma_{k} m_{k}\right], \quad D_{k}^{G}=D_{k}^{F}-\frac{\Omega}{4 D \beta_{6}{ }^{2}} \rho_{n} \sigma_{n}{ }^{2} \sigma_{k} m_{k}, \\
\Omega=\sum_{k=1}^{n} \rho_{k} \sigma_{k} m_{k} D_{k}^{F}, \\
D_{a}=\frac{\beta_{6}{ }^{2}}{4}\left\{\sum_{k=1}^{n} \rho_{k}\left(N_{k} \sigma_{k}+Z_{k}\right)^{2}+\frac{1}{4} \rho_{n} \sigma_{n}{ }^{2}\left[\frac{1}{9}\left(\sigma_{n} B\right)^{2}-\frac{\omega^{2}}{D}\right]\right\} \\
\omega=\frac{2}{\beta_{6}{ }^{2}} \Omega+\frac{1}{3} \sigma_{n} B D .
\end{gathered}
$$


It must be underlined that Eqs. (9) and (13) now include the new parameters $N_{n}$ and $m_{n}$, respectively. According to Eqs. (53) and (56) of B0, or to Eqs. (1.26) and (1.40) of BW1, $N_{i}$ and $m_{i}$ account for the interaction of ion $i$ with other ions, and with the solvent, respectively. Consequently, $n$ is again viewed as a solute particle in the parameters $N_{n}$ and $m_{n}$.

It is also noted that Eq. (13) for $D_{a}$ differs from the one given in BW1, BW2, and S0, which involves an inconvenient summation. Eq. (13) was obtained from Eqs. (60) and (B35) of B0, and it was verified that it is equivalent to the previous expression.

In Eqs. (2)-(4), $K_{i j}^{m p}$ is given by,

$$
K_{i j}^{m p} \equiv \int_{\lambda_{j i}}^{\sigma_{i j}} Q_{i j}^{m p}(r) d r
$$

in which

$$
\lambda_{j i}=\left(\sigma_{j}-\sigma_{i}\right) / 2
$$

and $Q_{i j}^{m p}$ is the Baxter factor correlation function,

$$
Q_{i j}^{m p}(r)=\frac{1}{2}\left(r-\sigma_{i j}\right)\left(r-\lambda_{j i}\right) \alpha_{j}^{m p}+\left(r-\sigma_{i j}\right) \beta_{i j}^{m p},
$$

in which $\alpha_{j}^{m p}$ (三 $\left.\alpha_{i j}^{m p}\right)$ does not depend on $i$ (see BW1). By using Eqs. (34) and (43) of S0, it is found that the parameter $\alpha_{j}^{00}$ may be expressed as,

$$
\alpha_{j}^{00}=\alpha_{j}^{00, H S}+\alpha_{j}^{00, e l}
$$

where

$$
\alpha_{j}^{00, H S}=L\left(2+L \xi_{2} \sigma_{j}\right),
$$

is the hard sphere (HS) part of $\alpha_{j}^{00}$, that is the value of this parameter when all charges on the ions are taken away, and Eq. (43) of S0 can be simplified to give,

$$
\alpha_{j}^{00, e l}=-\frac{1}{D \beta_{6}} \rho_{n} \sigma_{n}^{2} S_{m} \eta_{j}+\frac{2 L}{\beta_{6}} S_{G} a_{j}^{0},
$$

for the purely electrostatic part. For the ion-dipole interaction one gets,

$$
\alpha_{n}^{01}=-2 \frac{\beta_{3}}{\beta_{6}{ }^{2}} \frac{S_{m}}{D}+\frac{2 L}{\beta_{6}} S_{G} a_{n}^{1}, \quad \alpha_{j}^{10}=\frac{b_{2}}{\beta_{6} \sigma_{n}} \beta_{n j}^{10}+\frac{1}{\beta_{6}} B a_{j}^{0},
$$

in which $\alpha_{n}^{01}$ has been simplified, and $j$ may designate an ion or the solvent. For the dipoledipole interaction one has,

$$
\alpha_{n}^{11}=2 \frac{b_{2}}{\beta_{6} \rho_{n} \sigma_{n}^{3}}\left(1+\frac{1}{2} \rho_{n} \sigma_{n}{ }^{2} \beta_{n n}^{11}\right)+\frac{1}{\beta_{6}} B a_{n}^{1},
$$


For the $\beta$ 's one has, as in S0,

$$
\beta_{i j}^{00}=\beta_{i j}^{00, H S}+\beta_{i j}^{00, e l}
$$

where,

$$
\beta_{i j}^{00, H S}=L \sigma_{j}, \quad \beta_{i j}^{00, e l}=-\frac{1}{2 D \beta_{6}} \rho_{n} \sigma_{n}{ }^{2} m_{i} \eta_{j}+\frac{1}{\sigma_{i}}\left(\frac{2}{\beta_{6}} D_{i}^{G}-Z_{i}\right) a_{j}^{0}
$$

and,

$$
\beta_{i n}^{01}=-\frac{\beta_{3}}{\beta_{6}{ }^{2}} \frac{m_{i}}{D}+\left(\frac{2}{\beta_{6}} D_{i}^{G}-Z_{i}\right) \frac{1}{\sigma_{i}} a_{n}^{1}, \quad \beta_{n j}^{10}=\frac{1}{D}\left(\eta_{j}+\frac{1}{\beta_{6}{ }^{2}} \Omega a_{j}^{0}\right),
$$

in which $i$ and $j$ may designate ions or a dipolar solvent particle, and,

$$
1+\frac{1}{2} \rho_{n} \sigma_{n}{ }^{2} \beta_{n n}^{11}=\frac{1}{D}\left(\frac{\beta_{3}}{\beta_{6}}+\frac{1}{2 \beta_{6}{ }^{2}} \rho_{n} \sigma_{n}{ }^{2} \Omega a_{n}^{1}\right),
$$

We will need the quantity $P_{i j}^{m p}$ (with $m$ and $p=0$ or 1 ), which is defined by (see S0),

$$
P_{i j}^{m p}=\sigma_{i} \beta_{i j}^{m p}+Z_{i} a_{j}^{p},
$$

for any $i, j=1, \ldots, n$. The $P$ 's satisfy the symmetry relations (see S0),

$$
P_{i j}^{m p}=P_{j i}^{p m}
$$

This property will be used below to determine the numerical values of the MSA parameters of the model.

Moreover, it was found in this work that the $P$ 's now should fulfill the following slightly modified 'sum rules' (see also Section IV D below),

$$
D_{j}^{F}+\frac{1}{2} \sigma_{j} \sum_{k=1}^{n} \rho_{k} P_{k j}^{00} D_{k}^{F}=\frac{1}{2} Z_{j} \beta_{6},
$$

for $j=1, \ldots, n$, and,

$$
\sum_{k=1}^{n} \rho_{k} P_{k n}^{01} D_{k}^{F}=\frac{1}{2} \sigma_{n} B
$$

in which the summations are extended to include $k=n$.

\section{A. Dimensionless energy parameters}

As in S0, dimensionless parameters are introduced to replace the $N_{i}{ }^{\prime}$ 's and the $m_{i}$ 's. For the ions, this may be done, for example as in S0, by setting,

$$
N_{k}=\frac{Z_{k}}{\sigma_{k}} b_{0}^{(k)}, \quad m_{k}=\frac{1}{\sqrt{\eta \rho_{n}} \sigma_{n}} \frac{Z_{k}}{\sigma_{k}} b_{1}^{(k)},
$$


with $\eta=\sum \rho_{k} Z_{k}^{2}$. Eq. (31) involves a parameter $b_{0}^{(k)}$ for the interaction of $k$ with the other ions and the neutral solute $n$, and a parameter $b_{1}^{(k)}$ for the interaction of $k$ with the solvent. With these definitions, one has $b_{0}^{(k)}<0$ and $b_{1}^{(k)}>0$ for all ions.

In the case of the solvent $n$, since $Z_{n}=0$, one may introduce the parameters $b_{0}^{(n)}$ and $b_{1}^{(n)}$ as,

$$
N_{n}=\frac{1}{\sigma_{n}} b_{0}^{(n)}, \quad m_{n}=\frac{1}{\sqrt{\eta \rho_{n}} \sigma_{n}^{2}} b_{1}^{(n)}
$$

Because particle $n$ is regarded as an electrically neutral solute, and the parameters $b_{0}^{(k)}$ and $b_{1}^{(k)}$ account for the electrostatic interactions of particle $k$ with the ions and the solvent, respectively, it is expected that the order of magnitude of $b_{0}^{(n)}$ and $b_{1}^{(n)}$ should be appreciably lower than those for the charged ions.

These parameters $b_{0}^{(k)}$ and $b_{1}^{(k)}$ for $k=1, \ldots n$, together with $b_{2}$ (which quantifies the solvent-solvent interactions), are the unknowns to determine in the first place when performing numerical evaluations for a particular system. The thermodynamic quantities are expressed in terms of these parameters.

In the general case, one has $n(n-1) / 2$ equations for the symmetry of the $P_{i j}^{00}$ 's with $i$ and $j$ in the range of 1 to $n,(n-1)$ equations for the $P_{i n}^{01}$, and the 3 fundamental equations (2)-(4). This makes a total of $n(n+1) / 2+2$ equations for $(2 n+1)$ parameters $\left[b_{0}^{(k)}, b_{1}^{(k)}\right.$, with $k=1, \ldots, n$, and $\left.b_{2}\right]$ to be determined. The difference between the two is $n(n-3) / 2+1$ which is always positive because $n \geq 3$. There are therefore more equations than unknowns. It is expected that $n(n-3) / 2+1$ relations may be derived from the $(2 n+1)$ equations chosen to determine the parameter values.

\section{THERMODYNAMIC PROPERTIES}

\section{A. Pressure and internal energy}

The expression for the pressure that was given in S0 must be completed by introducing also the effect of the solvent being viewed as a neutral solute. One has ${ }^{5}$,

$$
\beta P^{e l}=J+J^{\prime},
$$


in which $J$ and $J^{\prime}$ are given by

$$
\begin{gathered}
J=\frac{1}{12 \pi}\left(\alpha_{0}^{2} \sum_{i} \rho_{i} Z_{i} N_{i}-4 \alpha_{0} \alpha_{2} \rho_{n} B-\frac{6}{{\sigma_{n}}^{3}} \alpha_{2}^{2} \rho_{n} b_{2}\right), \\
J^{\prime}=J^{\prime}{ }_{\text {ion-ion }}+2{J^{\prime}}^{\prime}{ }_{\text {ion }-n}+J^{\prime}{ }_{n-n},
\end{gathered}
$$

where,

$$
\begin{gathered}
J^{\prime}{ }_{i o n-i o n}=\frac{\pi}{3} \sum_{\{i, j\}=1}^{n} \rho_{i} \rho_{j}\left(\sigma_{i j}\right)^{3}\left[\left(g_{i j, c}^{000}\right)^{2}-\left(g_{i j, c}^{H S, 000}\right)^{2}\right], \\
J^{\prime}{ }_{i o n-n}=\frac{\pi}{3} \sum_{i=1}^{n} \rho_{i} \rho_{n}\left(\sigma_{i n}\right)^{3} \frac{1}{3}\left(h_{i n, c}^{011}\right)^{2},
\end{gathered}
$$

where the summations have been extended to $i$ and $j=n$, and,

$$
J_{n-n}^{\prime}=\frac{1}{12 \pi}\left(\rho_{n}\right)^{2} \sigma_{n}\left[\left(Q_{n n}^{\prime 11}\right)^{2}+2 q^{\prime 2}\right] .
$$

In these relations, $g_{i j, c}^{000}$ is the component of the contact RDF (radial distribution function) along the rotational invariant, $\Phi^{000}=1$. The expressions for the contact RDF's, $g_{i j, c}^{000}, g_{i j, c}^{H S}$, $h_{i n, c}^{011}$, and for $Q_{n n}^{\prime 11}$ and $q^{\prime}$, were given in S0, and they are still valid.

The expression for the electrostatic contribution to the internal energy is unchanged,

$$
\frac{\beta E}{V}=\frac{1}{4 \pi}\left(\alpha_{0}^{2} \sum_{i=1}^{n-1} \rho_{i} Z_{i} N_{i}-2 \alpha_{0} \alpha_{2} \rho_{n} B-2 \alpha_{2}^{2} \frac{1}{\sigma_{n}{ }^{3}} \rho_{n} b_{2}\right) .
$$

with $V$ the volume. This relation stems directly from the definition of the internal energy in terms of the RDF's (see BW1).

\section{B. Determination of the chemical potentials}

As emphasized in S0, the electrostatic contribution to the chemical potential of a species may be written as,

$$
\mu_{k}=u_{k}+\delta \mu_{k}
$$

where $u_{k}$ is the mean electrostatic interaction energy per particle $k$ (= ion or solvent), given by,

$$
\beta u_{i}=Z_{i} \frac{1}{4 \pi}\left(\alpha_{0}^{2} N_{i}-\alpha_{0} \alpha_{2} \rho_{n} m_{i}\right)
$$

for the ions, and,

$$
\beta u_{n}=-\frac{1}{4 \pi}\left(\alpha_{0} \alpha_{2} B+2 \alpha_{2}^{2} \frac{1}{\sigma_{n}^{3}} b_{2}\right) .
$$


for the solvent.

The contribution $\delta \mu_{k}$ will now be determined in the next two sections, first for the ions and then for the solvent.

\section{Calculation of $\delta \mu_{k}$ for the ions}

In the case of the ions, we employed a method similar to one of those proposed in the absence of dipoles ${ }^{10}$. This method is based on the work of Høye and Stell ${ }^{12}$ which yields ${ }^{10}$,

$$
\beta \delta \mu_{i}=\beta u_{i}-\frac{1}{2}\left\langle\left[c_{i i}(0)-c_{i i}^{H S}(0)\right]\right\rangle,
$$

in which the brackets indicate an average over the orientations, and $c_{i i}(0)$ is the value of the $i-i$ direct correlation function, $c_{i i}(r)$, at $r=0$.

When $i$ is an ion one has,

$$
\beta \delta \mu_{i}=\beta u_{i}-\frac{1}{2}\left[c_{i i}^{000}(0)-c_{i i}^{000, H S}(0)\right],
$$

because only the $c_{i i}^{000}(0)$ contribution along the rotational invariant $\Phi^{000}=1$ remains for the ions. This quantity may be found from the following relation,

$$
c_{i i}^{000}(0)=-\left.\frac{1}{2 \pi} \frac{\mathrm{d}^{2} S_{i i, 0}^{00}(r)}{\mathrm{d} r^{2}}\right|_{r=0}
$$

which ensues from the definition of $S_{i j, 0}^{00}$ given in Eq. (13) of B0 (employed with $\chi=l=0$ in the present case),

$$
S_{i j, 0}^{00}(r)=2 \pi \int_{r}^{\infty} t c_{i j}^{000}(t) \mathrm{d} t
$$

which may be obtained from a formula derived in B0. It may be written in a more simple form for $j=i$ as $^{10}$,

$$
S_{i i, 0}^{00}(r)=Q_{i i}^{00}(r)-A_{i i}^{00}-\sum_{p=0,1} I_{i p}(r)
$$

with

$$
I_{i p}(r)=\sum_{k=1}^{n} \rho_{k}\left[\int_{\lambda_{k i}}^{\sigma_{i k}-r} Q_{i k}^{0 p}(r+t) Q_{i k}^{0 p}(t) \mathrm{d} t-A_{i k}^{0 p} \int_{\lambda_{k i}+r}^{\sigma_{i k}} Q_{i k}^{0 p}(t) \mathrm{d} t-A_{i k}^{0 p} \int_{\lambda_{k i}}^{\sigma_{i k}} Q_{i k}^{0 p}(t) \mathrm{d} t\right]
$$

where

$$
A_{i k}^{0 p}=Z_{i} a_{k}^{p}
$$


In Eq. (48), the case $p=1$ imposes that $k$ can only take the value $k=n$, because $Q_{i k}^{01}(r)=0$ and $A_{i k}^{01}=0$ when $k$ is not a solvent particle. Following Ref. 10, and using Eqs. (45), (47), and (48), one gets,

$$
c_{i i}^{000}(0)=\frac{1}{2 \pi}\left\{-\alpha_{i}^{00}+\sum_{k=1}^{n} \rho_{k}\left[K_{i k}^{00} \alpha_{k}^{00}+\left.A_{i k}^{00} \frac{d Q_{i k}^{00}(r)}{d r}\right|_{r=\lambda_{k i}}\right]+\rho_{n}\left[K_{i n}^{01} \alpha_{n}^{01}+\left.A_{i n}^{01} \frac{d Q_{i n}^{01}(r)}{d r}\right|_{r=\lambda_{n i}}\right]\right\}
$$

in which one has from Eqs. (16) and (17),

$$
\left.\frac{d Q_{i k}^{0 p}(r)}{d r}\right|_{r=\lambda_{k i}}=-\frac{1}{2} \sigma_{i} \alpha_{k}^{0 p}+\beta_{i k}^{0 p}
$$

In Eq. (50), all terms may be calculated from the definitions given in Section III. The calculation is rather straightforward but it turns out to be cumbersome, and the resulting expression is rather large. The algebraic computation system Maple was utilized to handle the calculation. The formula for $\delta \mu_{i}$ is displayed in the supplementary material. Attempts to simplify it were unsuccessful.

\section{Calculation of $\delta \mu_{n}$ for the solvent}

In the case of the solvent, the calculation of $\delta \mu_{n}$ from Eq. (43) is much more delicate than in the case of the ions, because it would require the derivation of $c_{n n}(0)$, which seemed very difficult.

Indeed, it was realized that $c_{n n}(0)$ contains the terms $c_{n n}^{110}(0)$ and $c_{n n}^{112}(0)$. Upon orientation averaging, the $c_{n n}^{112}(0)$ vanishes (the average of the $\Phi^{112}$ rotational invariant is zero for all $r$ ), but the average of $c_{n n}^{110}(0)$ does not. In the notation of Wertheim ${ }^{13}$, the $c_{n n}^{110}(0)$ term has the form: $c_{n n}^{110}(0)=c_{\Delta}(0) \mathbf{s}_{1} \cdot \mathbf{s}_{2}$, where $\mathbf{s}_{i}$ is a unit vector in the direction of the dipole moment of particle $i^{13,14}$. The evaluation of $c_{n n}^{110}$ at $r=0$ means that positions 1 and 2 are the same. Thus, upon averaging over orientations one gets, $c_{n n}^{110}(0)=c_{\Delta}(0)\left\langle\mathbf{s}_{1} \cdot \mathbf{s}_{1}\right\rangle=c_{\Delta}(0)\left\langle s_{i}^{2}\right\rangle=c_{\Delta}(0)$, and the latter is not easy to determine.

Instead, to circumvent the latter difficulty, the following formula from the work of Høye and Stell ${ }^{12}$ was considered,

$$
\beta \delta \mu_{j}=-\frac{1}{2} \sum_{i=1}^{n} \rho_{j}\left[\tilde{c}_{i j}^{000}(0)-\tilde{c}_{i j}^{000, H S}(0)\right]
$$


where $i$ and $j$ may represent any species (ion or solvent), and $\tilde{c}_{i j}^{000}(0)$ denotes the Fourier transform of $c_{i j}^{000}(r)$, in 3 dimensions over the space coordinate $\mathbf{r}$, and taken at $k=0$.

Let us note that Eq. (52) should also contain terms with dipolar orientations. But in this case the dipole moments $\mathbf{s}_{1}$ and $\mathbf{s}_{2}$ (in the notation of Wertheim) are different and, by averaging, terms with one or both of these dipole moments present will vanish.

Now, the purpose of this section is to show that $\tilde{c}_{i n}^{000}(0)$, and therefore $\delta \mu_{n}$, can be obtained from the results of the preceding section. This may be proven as follows.

For the dipolar hard spheres problem it is known that the dipolar part of its MSA solution decouples from the reference system of hard spheres alone ${ }^{13,14}$. Furthermore, one will find that dipole moments can be redistributed among the hard spheres with equal diameters while the solution remains the same ${ }^{15}$. Reasons for this property are that the dipole interaction averaged over orientations is zero and that the core condition upon the dipolar part of the correlation function remains zero inside hard cores. In view of this there is reason to expect that something similar is the situation for the MSA electrolyte. Also for the latter the average of the interactions over dipolar orientations is zero, also with ionic charges present. Further the dipolar part of the correlation function must be zero inside hard cores.

In section IV B 3 below we analyze the OZ (Ornstein-Zernike) equation for the MSA problem of the ion-dipole mixture. By evaluations connected to a graph expansion of the solution, we find the decoupling of interest. Thus dipole moments can be redistributed among particles having the same hard core diameter while the MSA solution must remain the same (except for trivial adjustments). Then a fraction of these particles may be left with no dipole moments, i.e. they have become equivalent to ions, but with zero charge (in this case where the dipolar particles have no charge). This again implies that Eq. (50) for $c_{i i}^{000}(0)$ is also valid for these new zero charge ions. Further, with unchanged MSA solution this equation is also valid for the particles left with the redistributed dipole moments. This follows from the analysis of the OZ equation and the results obtained as given by Eqs. (S33) and (74). The reason is that with Eq. (52) and the same MSA solution, there can be no difference between particles with or without dipole moments after the redistribution. Altogether, also for the dipolar solvent, the correction $\delta \mu_{i}$ follows from Eqs. (44) and (50). Thus the $c_{n n}^{110}(0)$ contribution mentioned above must cancel against the corresponding dipolar internal energy contribution. This follows from the MSA internal energy that is a combination of Eqs. (44) and (52). By its extension to the dipolar part of the energy, Eq. (52) does not contribute 
due to averaging over dipolar orientations.

\section{Graph expansion of the MSA solution for the ion-dipole mixture}

In this section we will show how the dipole moments of the solvent particles can be redistributed while the non-trivial part of the MSA solution remains the same. By redistribution, a part of the solvent particles may be left with no dipole moments. A consequence of this is that the correction $\delta \mu_{n}$ to the chemical potential of the solvent can be identified with the one for ions. With zero net charge on the dipolar particles the $\delta \mu_{n}$ will be the same as the one for ions with zero charge and same diameter.

For the dipolar solvent alone the possibility of redistribution of dipole moments was found already in Ref. 15. Since the dipolar part of this MSA problem separates from its hard sphere part alone, such redistribution may not be unexpected. However, for dipolar spheres in a mixture with charged particles this is far from obvious since charge-dipole interactions are involved. Thus we will show this in more detail by studying properties of the solution of the $\mathrm{OZ}$ equation for the ion-dipole mixture.

The OZ (Ornstein-Zernike) equation Fourier transformed is

$$
\tilde{h}=\tilde{c}+\tilde{h} \rho \tilde{c}
$$

with $\tilde{h}=\tilde{h}(k)$ and $\tilde{c}=\tilde{c}(k)$ where $k$ is the Fourier variable. This can be extended to mixtures as,

$$
\tilde{h}_{i j}=\tilde{c}_{i j}+\sum_{l} \tilde{h}_{i l} \rho_{l} \tilde{c}_{l j}
$$

Eq. (54) can be written in the notation of Eq. (53) when the quantities there are interpreted as matrices. It is convenient to include the "self-correlation" $\rho_{i} \delta_{i j}$ to get the quantity

$$
\tilde{S}_{i j}=\rho_{i} \delta_{i j}+\rho_{i} \tilde{h}_{i j} \rho_{j}
$$

by which Eq. (54) becomes

$$
\tilde{S}_{i j}=\rho_{i} \delta_{i j}+\sum_{l} \tilde{S}_{i l} \tilde{c}_{l j} \rho_{j}
$$

To obtain full matrix notation we further introduce

$$
\tilde{S}_{i j}=\rho_{i}^{1 / 2} \tilde{U}_{i j} \rho_{j}^{1 / 2} \text { and } \quad \tilde{c}_{i j}=\rho_{i}^{-1 / 2} \tilde{V}_{i j} \rho_{j}^{-1 / 2}
$$


by which Eq. (56) turns into

$$
\tilde{U}_{i j}=\delta_{i j}+\sum_{l} \tilde{U}_{i l} \tilde{V}_{l j}
$$

or in matrix notation

$$
\tilde{U}=I+\tilde{U} \tilde{V}
$$

where $I$ is the unit matrix. Solved with respect to $\tilde{U}$, this gives

$$
\tilde{U}=\frac{I}{I-\tilde{V}}=I+\tilde{V}+\tilde{V}^{2}+\tilde{V}^{3}+\cdots,
$$

or,

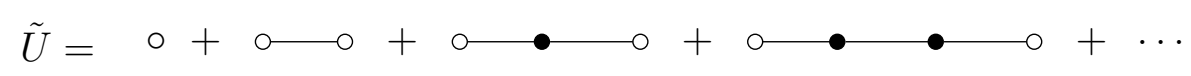

Here the graphs represent the solution in $r$ space where convolutions with $V=V(r)$ factors are performed.

For electrolytes the $\tilde{V}$ may be split in two parts

$$
\tilde{V}=\tilde{V}_{0}+\tilde{V}_{1}, \quad \tilde{V}_{1}=\tilde{V}_{C D}+\tilde{V}_{D D}
$$

The $V_{0}$ is taken as a reference situation and contains the charge-charge interactions and influence from the hard core condition. The $\tilde{V}_{1}$ contains the dependence on orientations from the dipole moments of the charge-dipole and dipole-dipole interactions, respectively.

With $\tilde{V}_{0}$ alone the solution is

$$
\tilde{U}_{0}=\frac{I}{I-\tilde{V}_{0}}
$$

When inserting for $\tilde{V}$ in Eq. (60) this gives

$$
\begin{gathered}
\tilde{U}\left(I-\tilde{V}_{0}-\tilde{V}_{1}\right)=\tilde{U}\left(I-\tilde{V}_{1} \tilde{U}_{0}\right) \tilde{U}_{0}^{-1}=I, \\
\tilde{U}_{0}=\tilde{U}\left(I-\tilde{V}_{1} \tilde{U}_{0}\right) .
\end{gathered}
$$

The solution of this equation is like expression (60), and it can be expanded in terms of graphs

$$
\tilde{U}=\tilde{U}_{0} \frac{I}{I-\tilde{V}_{1} \tilde{U}_{0}}
$$

or,

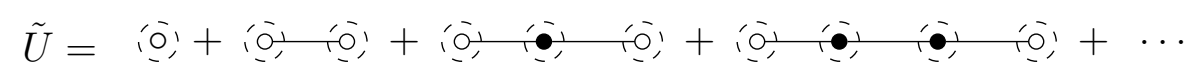


In this diagrammatic expansion, the open or filled symbols represent the $\tilde{U}_{0}$ instead of just the $I$ in Eq. (60). However, due to dipolar interactions, the situation is less straightforward in the sense that one has to integrate over orientations where dipole interactions are involved.

In the MSA the $\tilde{V}_{1}$ can have the following simplified and compact form

$$
\tilde{V}_{1}=s_{1} b_{2}+b_{1} s_{2}+c s_{1} s_{2}
$$

where the precise vectorial form has been omitted. This simplifies the analysis without loss of generality as long as the average of the one-dimensional substitute $s_{i}$ for the dipole moment is zero. Use of the simplified form compared to use of $\tilde{V}_{1}$ with charge-dipole and dipoledipole terms specified in detail, will not change the analysis. The first term in expression (67) represents the charge-dipole interaction between the dipole moment on particle 1 and the charge on particle 2. For the corresponding second term the charge of particle 1 and dipole moment of particle 2 are involved. Then the third term represents the dipole-dipole interaction between particles 1 and 2 . Here the one-dimensional substitute $s_{i}$ replaces the dipole moment vector $\mathbf{s}_{i}$. The crucial point is that the average of $s_{i}$ like the average of $\mathbf{s}_{i}$ over orientations is zero. [However, by looking more closely into this one finds that the dipole-dipole interaction splits into two terms that have factors with Fourier transforms $\left(\mathbf{k} \cdot \mathbf{s}_{1}\right)\left(\mathbf{k} \cdot \mathbf{s}_{2}\right)$ and $\left(\mathbf{k} \times \mathbf{s}_{1}\right)\left(\mathbf{k} \times \mathbf{s}_{2}\right)=\mathbf{s}_{1} \cdot \mathbf{s}_{2}-\left(\mathbf{k} \cdot \mathbf{s}_{1}\right)\left(\mathbf{k} \cdot \mathbf{s}_{2}\right)$. But the latter factor does not couple to the first one or the charge-dipole interaction $\left(\mathbf{k} \cdot \mathbf{s}_{1}\right)$ by integrations over $\mathbf{s}_{1}$ ].

For the resulting correlation function one now can use the corresponding simplified expression

$$
\tilde{U}=A+s_{1} B_{2}+B_{1} s_{2}+C s_{1} s_{2}
$$

Here we find it convenient to rename $\tilde{U}_{0}$ to be

$$
\tilde{U}_{0}=A_{0}
$$

The coefficients $B_{2}$ and $B_{1}$, like $b_{2}$ and $b_{1}$, are different since a pair of interacting particles have different charges and dipole moments. But these matrices are transpose of each other by which $B_{2}^{T}=B_{1}$ and $b_{2}^{T}=b_{1}$ (with $M_{i j}^{T}=M_{j i}$ ). The reason is that the term $s_{1} b_{2}$ represents the interaction between the dipole moment of particle 1 and the charge of particle 2 . The strength of their interaction is the matrix $b_{2}$ with elements $b_{2 i j}$ for a pair of particles of species $i$ and $j$. Likewise $s_{2} b_{1}$ is the corresponding interaction where particles 1 and 2 have changed roles. Thus, $b_{1 j i}=b_{2 i j}$, and then likewise with the matrices $B_{1}$ and $B_{2}$. [Here 
the general situation where any of the particle species may have both non-zero charge and dipole moment is not considered.]

With dipolar interactions, integrations have to be performed along with matrix multiplications. One has

$$
\left\langle s_{i}\right\rangle=\int s_{i} d s_{i}=0 \quad \text { and } \quad\left\langle s_{i}^{2}\right\rangle=\int s_{i}^{2} d s=1
$$

where $\left\langle s_{i}^{2}\right\rangle=1$ is used for simplicity.

Further, the form of $\tilde{U}$, and thus that of $A$ and $A_{0}$, is crucial by these integrations. From Eq. (58) one can write

$$
A=I+\Delta A, \quad A_{0}=I+\Delta A_{0}
$$

and with dependence upon orientations this means

$$
A=\delta(12) I+\Delta A, \quad A_{0}=\delta(12) I+\Delta A_{0}
$$

The $\delta(12)$ is a $\delta$-function in orientations at the same position in $r$ space. The second term is the regular pair correlation function that depends upon two different positions 1 and 2 , but does not vary with the two orientations.

Thus, with $\int d s_{1}=1$ and $\int s_{1} \delta(12) d s_{1}=s_{2}$ one finds the relations

$$
\int s_{1} A d s_{1}=\int s_{1} A_{0} d s_{1}=s_{2} I, \quad \text { and } \quad \int s_{2} A d s_{1}=A s_{2}, \quad \int s_{2} A_{0} d s_{1}=A_{0} s_{2} .
$$

Here in the first integral only the $\delta(12)$ function parts of $A$ and $A_{0}$ contribute. The $\Delta A$ and $\Delta A_{0}$ parts do not contribute since they do not depend upon orientations and $\left\langle s_{i}\right\rangle=$ 0 . However, in the second integral, both the $s_{2}, \Delta A$, and $\Delta A_{0}$ are merely constants of integration.

Now, the technical details needed to solve the $\mathrm{OZ}$ equation for the matrix coefficients $A$, $B_{1}, B_{2}$, and $C$ of the correlation function are given in the supplementary material. There, the solutions for these coefficients are analyzed and discussed in terms of graph expansions. From these expansions one finds that the dipole moments can be redistributed among hard spheres with the same diameter while the same MSA solution can be used.

As a consequence of Eq. (S33) for $k=0$, and by virtue of Eq. (52) employed for $j=n$ and $j=n_{0}$ separately, one obtains the simple result,

$$
\delta \mu_{n}=\delta \mu_{n_{0}}
$$


in which $\delta \mu_{n_{0}}$ may be calculated by using Eqs. (44) and (50) for the neutral solute $n_{0}$ that has the same size as the solvent, and from which the dipole moment has been removed $\left(d_{n_{0}}=0\right)$.

The result for $\delta \mu_{n}$ is therefore given by Eq. (S1) for $i=n_{0}$ (see the supplementary material). For convenience, the neutral solute $n_{0}$ was taken at a trace level (vanishingly small concentration), in which case the concentration of $n_{D}$ was that of the solvent $n$. In this way, the species $n_{0}$ does not need be considered in the search for the solution of the MSA-ID equations (as done below in Sec. V for the determination of $S_{b}$ ).

\section{Consequences of graph expansions: Generalization to more complex systems}

\section{General considerations}

An additional consequence of the graph expansions (S22)-(S30) is that the MSA solution with a dipolar solvent is valid and can be expanded to a more general situation as long as the dipolar particles all have the same hard core diameter. Thus the dipolar particles may be a mixture of particles that have different dipole moments, and they may also have different net charges.

The reason is that vertices where the interaction bonds $b_{1}, b_{2}$, and $c$ meet in the graphs for $D$ and $F$ in Eqs. (S24) and (S30) are just $I$. This means that interactions have to meet at the same point to give a contribution by integration over dipolar orientations. Thus only the unity matrix $I$ of the vertex function $A_{0}=I+\Delta A_{0}$ contributes. With Eq. (57), one has: $\tilde{V}_{i j}=\rho_{i}^{1 / 2} \tilde{c}_{i j} \rho_{j}^{1 / 2}$. For dipole-dipole interactions $\tilde{c}_{i j} \propto d_{D i} d_{D j}$ while for the charge-dipole one $\tilde{c}_{i j} \propto d_{D j}$, when particle $j$ is the dipole. Thus when a pair of dipole moments $d_{D_{j}}$ meet at a vertex, a factor or contribution $\rho_{j} d_{D_{j}}^{2}$ is created. For the MSA solution of the electrolyte with one dipolar component $d_{D_{n}}=d_{n}$ the factor $H=\rho_{n} d_{n}^{2}$ is produced at each such vertex.

With more polar components, contributions sum up, and this quantity is replaced by

$$
H=\rho_{n} d_{n}^{2}=\sum_{j} \rho_{j} d_{D_{j}}^{2}
$$

provided all components with non-zero dipole moments $d_{D_{j}}$ have the same hard core diameter $\sigma_{j}=\sigma_{n}$. This is also the same as the result obtained by Adelman and Deutch for the pure 
dipolar hard sphere fluid ${ }^{15}$. By that the dipole-dipole part of all $\tilde{c}_{i j}$, except for factors $d_{D_{i}} d_{D_{j}}$, will have the same $r$-dependence inside and outside the hard cores by which the MSA problem remains unchanged, and its solution can be directly utilized for this more general situation.

\section{Internal energies per particle}

In view of the preceding section, the internal energies per particle, $u_{i}$ (the $u_{n}$ included), of the various types of species can be expressed in one common expression.

To do so the $u_{i}$ (including $u_{n}$ ) are separated into two parts

$$
u_{i}=u_{i}^{(I)}+u_{i}^{(D)}
$$

The contributions $u_{i}^{(I)}$ and $u_{i}^{(D)}$ follow in a straightforward way from Eqs. (41) and (42). The first one is simply

$$
\beta u_{i}^{(I)}=\frac{Z_{i}}{4 \pi}\left(\alpha_{0}^{2} N_{i}-\alpha_{0} \alpha_{2} \rho_{n} m_{i}\right),
$$

So, the energies of the ionic charges remain unchanged. For $Z_{i}=0$, one has $u_{i}=0$. When the dipole moments are distributed over several components in accordance with Eq. (75), the $u_{i}^{(D)}$ becomes

$$
\beta u_{i}^{(D)}=-\frac{d_{D_{i}}^{2}}{4 \pi d_{n}^{2}}\left(\alpha_{0} \alpha_{2} B+2 \alpha_{2}^{2} \frac{1}{\sigma_{n}^{2}} b_{2}\right) .
$$

The resulting expressions for $u_{i}$ are valid when the dipole moments $d_{D_{i}}$ are non-zero only when the corresponding hard core diameters are such that $\sigma_{i}=\sigma_{n}$. With Eqs. (76)-(78) the internal energy per unit volume $E=\sum_{i} \rho_{i} u_{i}$ remains unchanged and is given by Eq. (39) when Eq. (75) is fulfilled.

\section{Thermodynamic consistency checks}

It was verified numerically within Maple, by using numbers with 50 digits, that the thermodynamic quantities fulfill some fundamental relations. This test was done in the case of a binary solution of a one molar 1-1 salt in a water-like solvent at $25^{\circ} \mathrm{C}$, by taking the following values for the parameters for the ions and the solvent ${ }^{16}: \sigma_{1}=2 \AA$ for the cation, $\sigma_{2}=4 \AA$ for the anion, $\sigma_{n} \simeq 2.4805 \AA$, and $d_{n} \simeq 2.2203 \mathrm{D}$. The density of the solvent was taken constant, equal to that of pure water. 
The thermodynamic relations are summarized in Table I together with the accuracy to which they are satisfied for values of a parameter $\varepsilon$ that represents the relative increment by which the variables are varied. For example, in Eq. (1), the derivative of $\beta P$ w.r.t. $\beta$ was obtained by computing $\beta P$ for 2 values of $\beta, \beta_{1}=\beta_{0}(1-\varepsilon)$ and $\beta_{2}=\beta_{0}(1+\varepsilon)$. The derivative was then approximated by $\left(\beta_{2} P_{2}-\beta_{1} P_{1}\right) /\left(\beta_{2}-\beta_{1}\right)$, with $P_{i}$ the value of $P$ for $\beta_{i}$.

In Table I, the Helmholtz energy $A$ was computed from the general relation, $A / V=$ $\sum_{k} \rho_{k} \mu_{k}-P$. The accuracies reported in this Table are the absolute values of the relative differences between the two sides of the relations. Fulfilment of the Gibbs-Duhem relation was tested at constant temperature by checking the relation in the following form,

$$
\frac{\partial P}{\partial \rho_{s}}+\rho_{s} \frac{\partial \mu_{s}}{\partial \rho_{s}}+\rho_{n} \frac{\partial \mu_{n}}{\partial \rho_{s}}=0
$$

in which subscript $s$ designates the salt.

TABLE I. Accuracies observed in the thermodynamic consistency checks.

\begin{tabular}{lrrc}
\hline Relation & $\varepsilon=10^{-2}$ & $\varepsilon=10^{-3}$ & $\varepsilon=10^{-4}$ \\
\hline$\frac{\partial \beta P}{\partial \beta}=-\frac{\partial E}{\partial V}$ & $6.10 \times 10^{-4}$ & $6.10 \times 10^{-6}$ & $6.10 \times 10^{-8}$ \\
$\mu_{s}=\frac{\partial(A / V)}{\partial \rho_{s}}$ & $3.59 \times 10^{-8}$ & $3.59 \times 10^{-10}$ & $3.59 \times 10^{-12}$ \\
$\mu_{n}=\frac{\partial(A / V)}{\partial \rho_{n}}$ & $3.72 \times 10^{-6}$ & $3.72 \times 10^{-8}$ & $3.72 \times 10^{-10}$ \\
$\frac{E}{V}=\frac{\partial(\beta A / V)}{\partial \beta}$ & $4.15 \times 10^{-6}$ & $4.15 \times 10^{-8}$ & $4.15 \times 10^{-10}$ \\
$\frac{\partial(E / V)}{\partial \rho_{s}}=\frac{\partial\left(\beta \mu_{s}\right)}{\partial \beta}$ & $1.28 \times 10^{-6}$ & $1.28 \times 10^{-8}$ & $1.28 \times 10^{-10}$ \\
$\frac{\partial(E / V)}{\partial \rho_{n}}=\frac{\partial\left(\beta \mu_{n}\right)}{\partial \beta}$ & $8.97 \times 10^{-7}$ & $8.97 \times 10^{-9}$ & $8.97 \times 10^{-11}$ \\
$\frac{\partial \mu_{s}}{\partial \rho_{n}}=\frac{\partial \mu_{n}}{\partial \rho_{s}}$ & $3.13 \times 10^{-5}$ & $3.13 \times 10^{-7}$ & $3.13 \times 10^{-9}$ \\
Gibbs-Duhem [Eq. (79)] $7.79 \times 10^{-7}$ & $7.79 \times 10^{-9}$ & $7.79 \times 10^{-11}$ \\
\hline
\end{tabular}

It is observed in Table I that the accuracies vary as $\varepsilon^{2}$ for a given relation. It is shown in the supplementary material that this is precisely the way they should vary when the relation is indeed valid analytically.

These numerical tests prove that the above equations for the thermodynamic quantities form a globally consistent set of relations, and that they provide the solution to the MSA-ID problem. 


\section{ILLUSTRATION IN THE CASE OF A BINARY 1-1 ELECTROLYTE}

We now specialize to the case of a binary 1-1 electrolyte in a water-like solvent at $25^{\circ} \mathrm{C}$ having the parameters employed in the preceding section: $\sigma_{1}=2 \AA$ (cation), $\sigma_{2}=4 \AA$ (anion), $\sigma_{n} \simeq 2.4805 \AA$, and $d_{n} \simeq 2.2203 \mathrm{D}$.

In this case, the unknowns are the set of parameters $S_{b}=\left\{b_{0}^{(1)}, b_{0}^{(2)}, b_{0}^{(n)}, b_{1}^{(1)}, b_{1}^{(2)}, b_{1}^{(n)}, b_{2}\right\}$. The values of these 7 parameters were determined numerically by again employing Maple, with numbers being expressed with 50 significant figures.

One has the 3 fundamental equations, Eqs. (2)-(4), and the five symmetry relations for $P_{i j}^{m p}$, Eq. (28),

$$
P_{12}^{00}=P_{21}^{00}, \quad P_{1 n}^{00}=P_{n 1}^{00}, \quad P_{2 n}^{00}=P_{n 2}^{00}, \quad P_{1 n}^{01}=P_{n 1}^{10}, \quad P_{2 n}^{01}=P_{n 2}^{10}
$$

Overall, this makes 8 equations and 7 unknowns in this particular case. It is expected that one of the equations may be derived from the others. In this example, it was found that it was preferable to leave the equation for $P_{1 n}^{00}$ aside. The same method as in $\mathrm{S} 0$ was used to numerically solve the equations and determine $S_{b}$. When the numerical solution for the set of parameters $S_{b}$ was obtained, it was verified that the equation for $P_{1 n}^{00}$ was indeed fulfilled.

The values of $b_{0}^{(1)}$ for the cation, and $b_{0}^{(2)}$ for the anion are plotted in Figure 2 as a function of the salt concentration $C$ (, together with the result obtained in $\mathrm{S} 0$ for these parameters, up to a concentration of $3 \mathrm{M}$. It is seen that the results for $b_{0}^{(1)}$ and $b_{0}^{(2)}$ are close to those found in S0.

The values of $b_{0}^{(n)}$ and $b_{1}^{(n)}$ are plotted in Figure 3. They turn out to be very small in comparison with those for the ions, as anticipated in Sec. III A because a solvent dipole carries a zero net charge.

The plots of $b_{1}^{(1)}$ and $b_{1}^{(2)}$ for the ions and those of $b_{2}$ are shown in the supplementary material, in Figures S1 and S2, respectively. These results for $b_{1}^{(1)}, b_{1}^{(2)}$, are also close to those of S0. The result for $b_{2}$ nearly coincides with that of S0.

The variation of the chemical potentials, and of the individual internal energies, of the ions and of the solvent, w.r.t. infinite dilution, are shown in Figure 4 by plotting $\Delta \mu_{i}$ and $\Delta u_{i}$, that are defined by

$$
\Delta \mu_{i}=\mu_{i}-\lim _{C \rightarrow 0} \mu_{i}, \quad \Delta u_{i}=u_{i}-\lim _{C \rightarrow 0} \mu_{i}
$$


in which it is noted that $\lim _{C \rightarrow 0} \mu_{i}=\lim _{C \rightarrow 0} u_{i}$. In the case of an ion, $\lim _{C \rightarrow 0} \mu_{i}$ is the electrostatic Gibbs solvation energy of the ion. The variation of the chemical potential of $i$ w.r.t. infinite dilution, $\Delta \mu_{i}$, also represents the electrostatic contribution to $\ln \gamma_{i}$ (with $\gamma_{i}$ the activity coefficient of $i$ ).

Figure 4 shows that the $\Delta \mu_{i}$ for the smaller ion (the cation here) is more negative than that for the bigger ion (the anion). Moreover, the individual internal energies $u_{i}$ are good approximations for the chemical potentials of the ions. It is also observed that $\beta \Delta \mu_{n}$ cannot be distinguished from $\beta \Delta u_{n}$ in the present case.

The relative magnitude of $\delta \mu_{i}$ was examined by plotting the ratio,

$$
R_{i}=\delta \mu_{i} / \Delta \mu_{i}
$$

as a function of the salt concentration $C$, where by virtue of Eqs. (40) and (81), one has

$$
\Delta \mu_{i}=\Delta u_{i}+\delta \mu_{i}
$$

The variation of $R_{i}$ for the ions is shown in Figure 5, and that of $R_{n}$ in Figure 6. It is seen in Figure 5 that $R_{i}$ is negative for the smaller ion (the cation here, $R_{1}<0$ ) and it is positive for the bigger (the anion, $R_{2}>0$ ). Moreover, their magnitude is of a few percent for this strongly asymmetric electrolyte, and $\left|R_{1}\right|<R_{2}$. It is noticed that these two outcomes are quite similar to what is found in the absence of dipoles (primitive model, see S0 and Ref. $10)$.

It is striking to observe in Figure 6 that $R_{n}$ is indeed very small. This confirms that $\delta \mu_{n}$ is much smaller than $\Delta u_{n}$ in Eq. (83) as seen in Figure $4\left(\beta \Delta \mu_{n} \simeq \beta \Delta u_{n}\right)$.

It was also found that the 'sum rules' given by Eqs. (29) and (30) were accurately fulfilled, with precisions better than $10^{-48}$.

\section{CONCLUSION}

In this work, the MSA for the ion-dipole mixture has been fully solved.

The results in a particular case at ambient temperature suggest that the chemical potentials of the ions are well approximated by their individual internal energies.

The expressions derived in this study for the chemical potentials of the ions and the solvent will be employed for a calculation of the mean salt activity and osmotic coefficients 
in aqueous electrolyte solutions. They will have to be computed at constant pressure $^{16}$ in order to fulfill the Gibbs-Duhem relation. A contribution arising from volume exclusion will be added to the present electrostatic one for their evaluation. It will also be attempted to include induced polarization of the solvent in the description.

In the field of chemical engineering applications, it will be possible to combine the present non-primitive unrestricted MSA-ID model with contributions that will account for effects such as ion-pairing, chemical association or hydrogen bonding, similarly to what has been done in the literature within the SAFT framework in conjunction with the non-primitive semi-restricted MSA-ID ${ }^{17,18}$.

\section{SUPPLEMENTARY MATERIAL}

See the supplementary material for the expression of $\delta \mu_{i}$ for the ions, technical details needed to solve the $\mathrm{OZ}$ equation, dependency of observed accuracy vs. value of increment $\varepsilon$ (section IV D), and plots of $b_{1}$ for the ions and of $b_{2}$.

\section{DATA AVAILABILITY}

The data that support the findings of this study are available from the corresponding author upon reasonable request.

\section{REFERENCES}

${ }^{1}$ L. Blum, "Solution of the mean spherical approximation for hard ions and dipoles of arbitrary size," J. Stat. Phys. 18, 451-474 (1978).

${ }^{2}$ L. Blum, "Invariant expansion III: The general solution of the mean spherical model for neutral spheres with electostatic interactions," J. Chem. Phys. 58, 3295-3303 (1973).

${ }^{3}$ L. Blum and D. Wei, "Analytical solution of the mean spherical approximation for an arbitrary mixture of ions in a dipolar solvent," J. Chem. Phys. 87, 555-565 (1987).

${ }^{4}$ D. Wei and L. Blum, "The mean spherical approximation for an arbitrary mixture of ions in a dipolar solvent: Approximate solution, pair correlation functions, and thermodynamics," J. Chem. Phys. 87, 2999-3007 (1987). 
${ }^{5}$ J.-P. Simonin, "On the solution of the mean-spherical approximation (MSA) for ions in a dipolar solvent in the general case," AIP Adv. 10, 095213 (2020), article in open access.

${ }^{6}$ L. Blum, F. Vericat, and W. Fawcett, "On the mean spherical approximation for hard ions and dipoles," J. Chem. Phys. 96, 3039-3044 (1992).

${ }^{7}$ L. Blum and A. J. Torruella, "Invariant expansion for two-body correlations: Thermodynamic functions, scattering, and the Ornstein-Zernike equation," J. Chem. Phys. 56, 303-310 (1972).

${ }^{8}$ L. Blum, "Invariant expansion. II. The Ornstein-Zernike equation for nonspherical molecules and an extended solution to the mean spherical model," J. Chem. Phys. 57, 1862-1869 (1972).

${ }^{9}$ L. Blum and J. Høye, "Mean spherical model for asymmetric electrolytes. 2. Thermodynamic properties and the pair correlation function," J. Phys. Chem. 81, 1311-1316 (1977).

${ }^{10}$ J. S. Høye and J.-P. Simonin, "Chemical potential of an ion in an asymmetric electrolyte within the mean spherical approximation (MSA)." Mol. Phys https://doi.org/10.1080/00268976.2021.1969045, in press.

${ }^{11}$ L. Blum, "Simple electrolytes in the mean spherical approximation," in Theoretical Chemistry, Advances and Perspectives, Vol. 5, edited by H. Eyring and D. Henderson (Academic Press: New York, 1980) pp. 1-66.

${ }^{12}$ J. S. Høye and G. Stell, "Thermodynamics of the MSA for simple fluids," J. Chem. Phys. 67, 439-445 (1977).

${ }^{13} \mathrm{M}$. Wertheim, "Exact solution of the mean spherical model for fluids of hard spheres with permanent electric dipole moments," J. Chem. Phys. 55, 4291-4298 (1971).

${ }^{14}$ J. Høye, J. Lebowitz, and G. Stell, "Generalized mean spherical approximations for polar and ionic fluids," J. Chem. Phys 61, 3253 (1974).

${ }^{15} \mathrm{~S}$. A. Adelman and J. M. Deutch, "Exact solution of the mean spherical model for simple polar mixtures," J. Chem. Phys. 59, 3971-3980 (1973).

${ }^{16}$ J.-P. Simonin, "On the "Born" term used in thermodynamic models for electrolytes," J. Chem. Phys. 150, 244503 (2019).

${ }^{17}$ W.-B. Liu, Y.-G. Li, and J.-F. Lu, "A new equation of state for real aqueous ionic fluids based on electrolyte perturbation theory, mean spherical approximation and statistical associating fluid theory," Fluid Phase Equilib. 158-160, 595 - 606 (1999).

${ }^{18} \mathrm{~S}$. Herzog, J. Gross, and W. Arlt, "Equation of state for aqueous electrolyte systems based 
on the semirestricted non-primitive mean spherical approximation," Fluid Phase Equilib. 297, 23 - 33 (2010). 


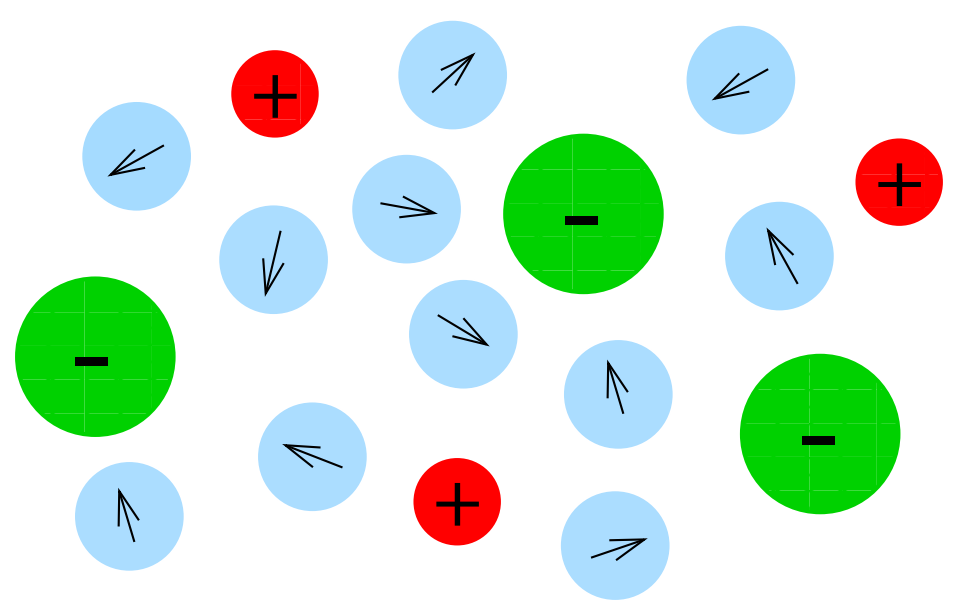

FIG. 1. Ion-dipole mixture. All species have different diameters.

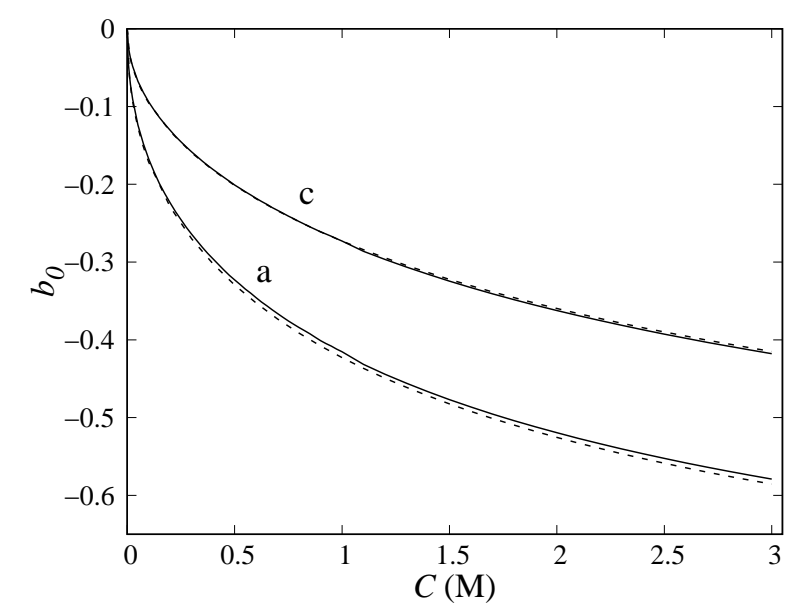

FIG. 2. Energy parameters $b_{0}$ for the ions as a function of $C$. Solid lines $=$ results of this work ("c" = cation, "a" = anion $)$, dashed lines = results obtained in S0. 


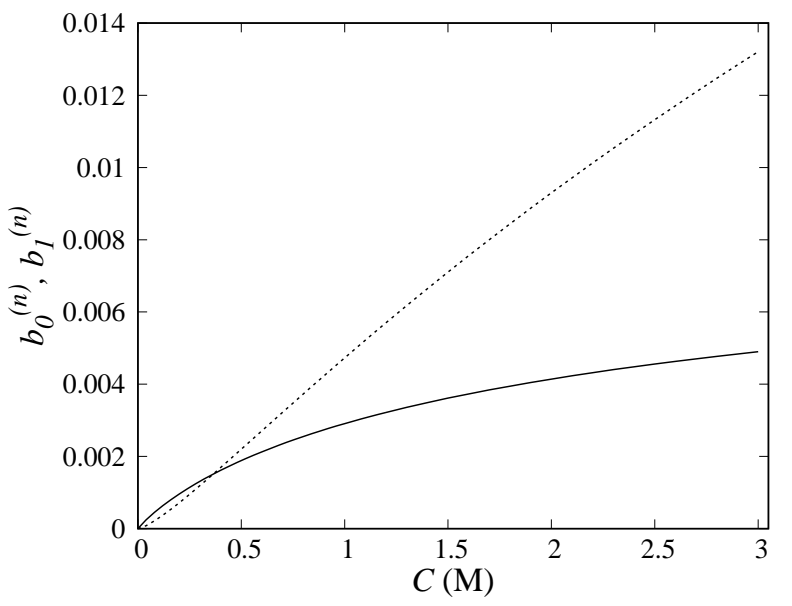

FIG. 3. Energy parameters for the solvent as a function of $C$. Solid line $=b_{0}^{(n)}$, dotted line $=b_{1}^{(n)}$.

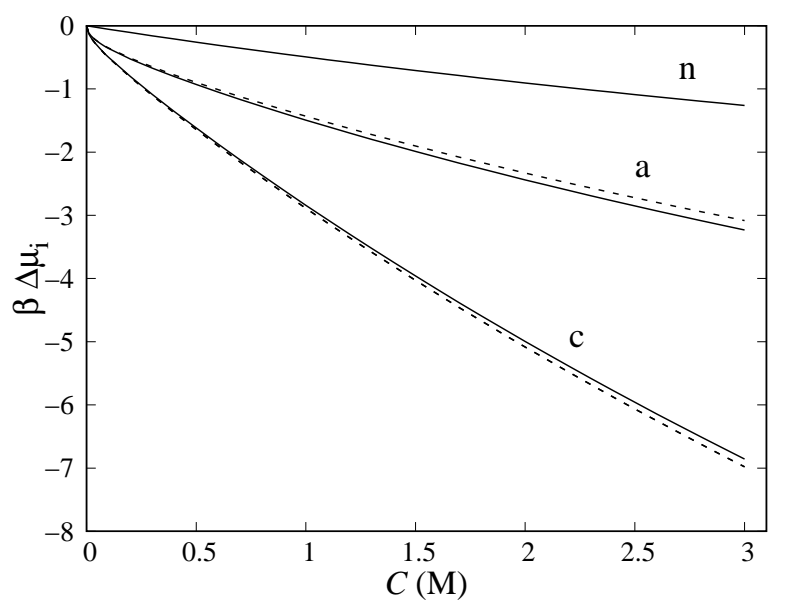

FIG. 4. Plot of $\beta \Delta \mu_{i}$ and $\beta \Delta u_{i}$. Solid lines $=\beta \Delta \mu_{i}$, dashed lines $=\beta \Delta u_{i}$; "c" = cation, "a" $=$ anion, "n"= solvent). 


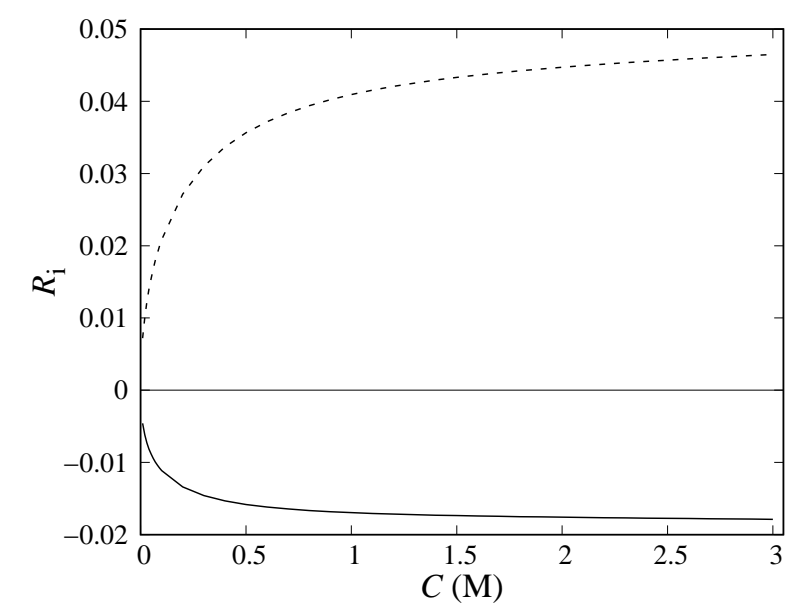

FIG. 5. Variation of $R_{i}$ for the ions as a function of the salt concentration, $C$. Solid line: cation; dashed line: anion.

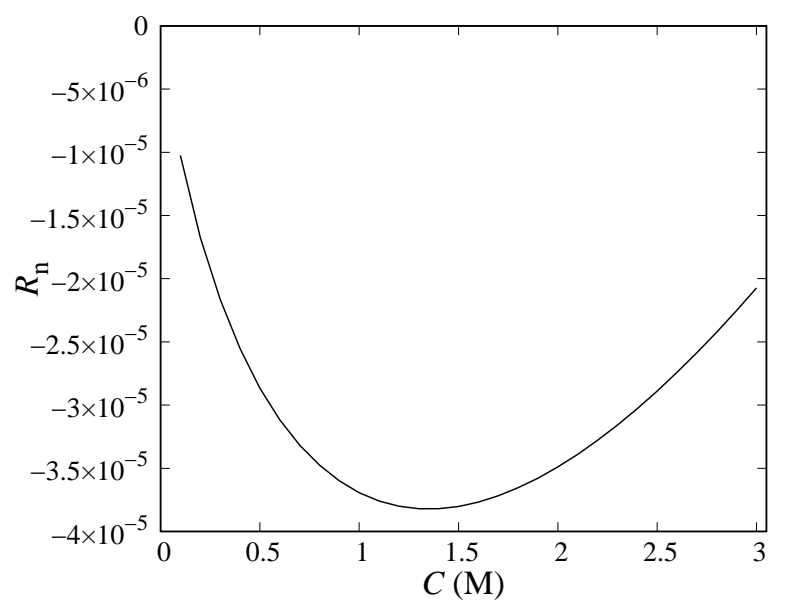

FIG. 6. Variation of $R_{n}$ for the solvent as a function of the salt concentration, $C$. 


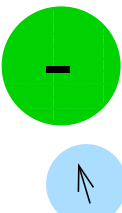

\section{1}

$K$

$$
\downarrow
$$

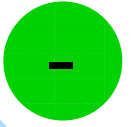

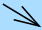
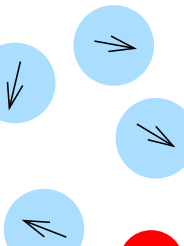

$\uparrow$

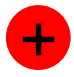

$\uparrow$

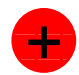

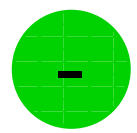

7 


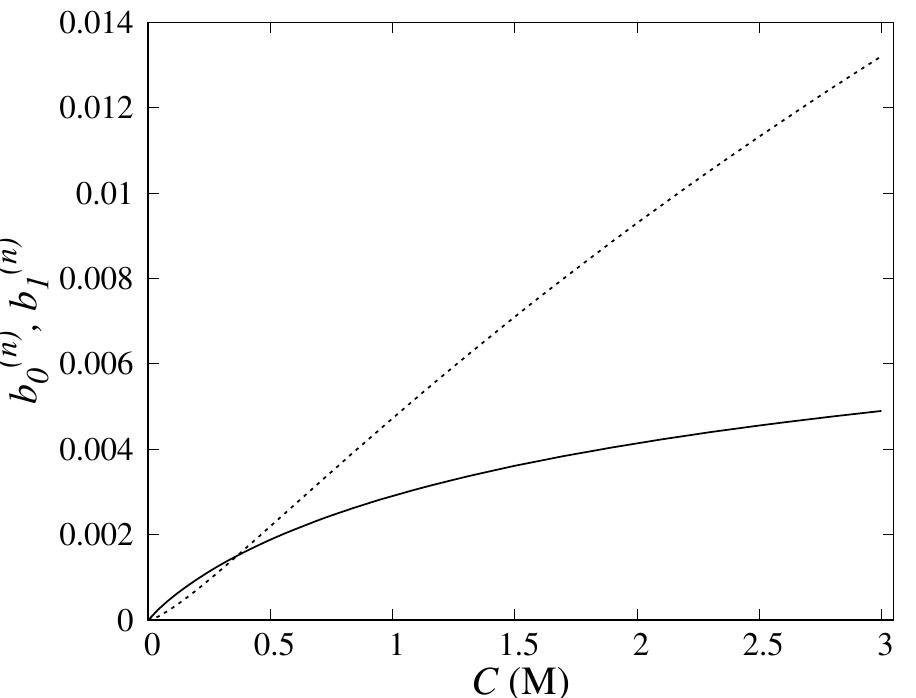


